\title{
The prevalence of occult ovarian cancer in the series of 155 consequently operated high risk asymptomatic patients - Slovenian population based study
}

\author{
Andreja Gornjec ${ }^{1}$, Sebastijan Merlo ${ }^{1}$, Srdjan Novakovic² ${ }^{2}$ Vida Stegel², Barbara Gazic ${ }^{3}$, \\ Andraz Perhavec ${ }^{4}$, Ana Blatnik ${ }^{5}$, Mateja Krajc ${ }^{5}$ \\ ${ }^{1}$ Department for Gynaecological Oncology, Institute of Oncology Ljubljana, Ljubljana, Slovenia
${ }^{2}$ Molecular Diagnostics Department, Institute of Oncology Ljubljana, Ljubljana, Slovenia
${ }^{3}$ Pathology Department, Institute of Oncology Ljubljana, Ljubljana, Slovenia
${ }^{4}$ Department for Surgical Oncology, Institute of Oncology Ljubljana, Ljubljana, Slovenia
${ }^{5}$ Cancer Genetics Clinic, Institute of Oncology Ljubljana, Ljubljana, Slovenia
}

Radiol Oncol 2020; 54(2): 180-186.

Received 11 December 2019

Accepted 22 March 2020

Correspondence to: Assist. Prof. Mateja Krajc, M.D., Ph.D., Cancer Genetics Clinic, Institute of Oncology Ljubljana, Zaloška cesta 2, SI-1000 Ljubljana, Slovenia. E-mail: mkrajc@onko-i.si

Disclosure: No potential conflicts of interest were disclosed.

Background. We assessed the prevalence, localization, type and outcome of occult cancer at risk-reducing salpingo-oophorectomy or salpingectomy (RRSO) in asymptomatic carriers of pathogenic or likely pathogenic BRCAl/2 variants and high-risk BRCAl/2 negative women.

Patients and methods. A retrospective analysis of all consecutive gynaecologic preventive surgeries from January 2009 to December 2015 was performed. Participants underwent genetic counselling and BRCAl/2 testing before the procedure. Data on clinical parameters, adjuvant treatment and follow-up were collected and analysed.

Results. One hundred and fifty-five RRSO were performed in 110 BRCA1, 35 BRCA2 carriers of pathogenic or likely pathogenic variants and 10 high-risk $B R C A l / 2$ negative women, at the mean age of 48.3 years. Nine occult cancers (9/155, 5.8\%) were identified; eight in BRCAl positive women and one in high-risk BRCAl/2 negative woman. We identified four non-invasive serous intraepithelial tubal carcinomas ( 3 in BRCAl carriers and 1 in a high-risk BRCAl/2 negative woman) and five invasive tubo-ovarian high grade serous cancers (all detected in BRCAl carriers). Only one out of nine patients (11.1\%) with occult cancer had a slightly elevated CA-125 value preoperatively.

Conclusions. A $5.8 \%$ prevalence of occult invasive and noninvasive tubo-ovarian serous cancer after RRSO was found in high risk asymptomatic and screen negative women. We conclude that RRSO should be performed in $B R C A l / 2$ carriers and in high-risk BRCAl/2 negative women. Age of preventive gynaecologic surgery should be carefully planned, taking into account the completion of childbearing age and type of mutation. The results favour the tubal hypothesis of tubal origin of high grade serous ovarian and peritoneal cancer. Cytology result of peritoneal cavity washing was important for the decision making process in determining treatment. Cytology examination should be performed in all cases of RRSO. CA-125 assay did not prove to be an effective screening tool for early cancer detection in our patients.

Key words: risk-reducing salpingo-oophorectomy (RRSO); occult serous cancer; serous tubal intraepithelial cancer (STIC); BRCAl/2 pathogenic or likely pathogenic variant 


\section{Introduction}

Worldwide, ovarian cancer is the seventh most common cancer and the eighth cause of death from cancer in women. ${ }^{1}$ According to the Slovenian cancer registry, the median age at the time of diagnosis for ovarian cancer patients is 62 years. $^{2}$ Epithelial ovarian cancer (EOC), being the commonest, is thought to be hereditary in at least $10 \%$ of cases, mostly due to BRCA1 or BRCA2 germline pathogenic or likely pathogenic variants. Ovarian cancer risk in BRCA carriers ranges from $36 \%-53 \%$ in $B R C A 1$ and $11 \%-25 \%$ in BRCA2 carriers by the age of 80 years, compared to $1-2 \%$ in the general population. ${ }^{3}$ In addition, it presents at a younger age than in patients without a genetic predisposition. Genetic counselling, testing and appropriate screening and preventive strategies can highly reduce the risk..$^{3-6}$

In more than $70 \%$, EOC is diagnosed in advanced stages (III/IV). This is mainly due to vague and non-specific symptoms, and because of an ineffective ovarian cancer screening. BRCA carriers are therefore recommended to undergo salpingooophorectomy or salpingectomy as a risk-reducing strategy for ovarian cancer., 3,7, A new paradigm of high grade pelvic serous cancer carcinogenesis puts fallopian tubes as an anatomic origin of the primary lesion. Salpingectomy with delayed oophorectomy might therefore be offered to $B R C A$ carriers younger than 40 (after given informed consent in a research setting) who have completed their reproduction. ${ }^{9,10}$

So far, no precancerous lesions, like intraepithelial carcinoma, have ever been found in the ovaries. Precancerous lesions were found only in fallopian tubes. The first report of precancerous lesions in fallopian tubes after risk-reducing salpingo-oophorectomy (RRSO) was as a non-invasive serous tubal intraepithelial carcinoma (STIC). ${ }^{11,12}$ Occult ovarian and tubal cancers where also found in specimens of $B R C A$ carriers after RRSO. Occult cancers have been reported to occur between $2 \%$ and $17 \%$ and STICs between $3 \%$ and $12 \%$, respectively. ${ }^{13-22}$ The occult cancer detection is probably influenced by the age at RRSO, gynaecological screening prior to RRSO, extent of the surgical removal of specimens and the accuracy of tubal pathohistological assessment. It is known that the protocol for sectioning and extensively examining the fimbriated end of the Fallopian tube (SEE-FIM) enables a more exact histopathological examination of the distal tube with its fimbrial part. ${ }^{23}$
Ovarian cancer risk reduction after RRSO is reported to be between $80 \%-96 \%$. The risk of primary peritoneal cancer after RRSO is between $1 \%-4 \% .{ }^{24}$, ${ }^{25}$ Recurrence rate of ovarian cancer after the diagnosis of an occult invasive carcinoma at RRSO is relatively high (16\%-47\%), despite predominantly early stage, and small volume disease. ${ }^{17,25}$ On the other hand, the STICs rarely recur as carcinoma $(5.8 \%-9 \%)$, and therefore chemotherapy may not be needed. ${ }^{24,26}$ Recommendations about the optimal treatment of STICs lesions remain unclear. Opinions for treatment of STIC lean toward staging procedures and observations, if no other lesions are found.

Our study aimed to assess the prevalence, localization, type and outcome of occult cancer at RRSO in asymptomatic carriers of pathogenic or likely pathogenic $B R C A 1 / 2$ variants and high-risk $B R C A 1 / 2$ negative women.

\section{Patients and methods}

All consecutively operated women (asymptomatic carriers of pathogenic or likely pathogenic $B R C A 1 / 2$ variants and high-risk BRCA1/2 negative) who underwent RRSO or salpingectomy from January 2009 to December 2015 at the Institute of Oncology Ljubljana, Slovenia, were included in our study.

From 1999 genetic counselling and testing is offered at the Institute for women with positive family history of ovarian and breast cancer. Women with confirmed $B R C A$ pathogenic or likely pathogenic variants and $B R C A$ negative women with high ovarian cancer risk (at least two first or second-degree relatives with ovarian cancer) are assessed in a multidisciplinary setting by an onco-genetic team. In accordance with the guidelines high risk women are advised to perform risk reducing procedures (RRSO). ${ }^{78,27}$ Until 2014, RRSO was offered only after women turned 40 years of age. From 2014 tubectomy with delayed oophorectomy is offered in a research setting to women younger than 40 years who have completed their reproduction.

Clinical data were retrospectively collected from women with occult ovarian/tube/peritoneal cancer or non-invasive high-grade serous intraepithelial tube carcinoma diagnosed at the time of RRSO. Collected data included age at RRSO, mutation status, type of mutation, preoperative cancer antigen 125 (CA-125) level, histopathology result, staging, treatment (surgery and adjuvant chemotherapy), 
recurrence rate, prior history or development of breast cancer after RRSO, disease status and vital status. Asymptomatic women with negative ovarian cancer screening test (normal CA-125 and gynaecological ultrasound) 6 months prior to RRSO were included. Patients with ovarian or tubal cancer diagnosis prior to RRSO and those whose RRSO was a part of breast cancer treatment were excluded from the analysis.

All RRSOs had the same surgical and pathological protocol. During surgery, before the salpingooophorectomy took place, peritoneal washing was performed and the material was sent for cytological examination. If there was no free fluid in the cavum Douglasi, we eluted the pelvis with $10 \mathrm{~mL}$ of physiologic solution and the material was sent for cytological examination. At our institution SEEFIM protocol is used for RRSO specimens. ${ }^{23}$ When occult cancer and STIC are diagnosed, staging procedure is offered. After staging procedure is performed, cancers are managed according to the national guidelines. ${ }^{7}$ In women with STIC, when no other lesion is found at staging procedure, only observation with regular follow up is offered. When malignant cells are found at cytological examination of peritoneal cavity washing with no other lesion at staging procedure, chemotherapy with carboplatin and paclitaxel is offered.

The start of follow-up after RRSO was defined as the date of RRSO, where no other treatment was required. After cancer treatment with chemotherapy, the start of follow-up was defined as the date of the last given chemotherapy. The end of followup was defined as the last outpatient visit at our institution.

The study was approved by the Ethical Committee of the Institute of Oncology Ljubljana (Number ERID-EK/15).

\section{Statistics}

Statistical analysis was performed using SPSS 22.0 for Windows. Descriptive statistics was used to describe the basic features of the data in the study. Differences between the groups were investigated with Student-t test. P-values $<0.05$ were considered to be statistically significant.

\section{Results}

In the period of our study (January 2009-December 2015), 155 women underwent RRSO due to high ovarian cancer risk.

\section{Characteristic of patients and RRSO procedures}

In our cohort, there were 110/155 (71.0\%) BRCA1 mutation carriers, 35/155 (22.6\%) BRCA2 mutation carriers and 10/155 (6.5\%) high-risk BRCA negative patients. A variant of uncertain significance (VUS) was detected in 4/10 high risk BRCA negative women.

Mean age at RRSO among our patients was 48.3 (29-72); 47.6 (29-72) for BRCA1 carriers and 49.1 (38-66) for BRCA2 carriers, the difference did not differ significantly $(\mathrm{p}=0.4)$. In high-risk $B R C A$ negative women mean age at RRSO was 52.2 (3664) years. Median age at RRSO was also assessed for further comparison with other studies and accounted 47 years.

Before RRSO was performed, 110/155 (71.0\%) women had already been diagnosed with breast cancer, 4/155 (2.6\%) women had the first breast cancer diagnosis before and the second breast cancer diagnosis after RRSO and 1/155 (0.6\%) woman was diagnosed with breast cancer after RRSO.

Among women where RRSO was performed, the mean age at breast cancer diagnosis was 54.0 (33-64) among women with STIC and 49.3 (38-61) among women with invasive cancer. The mean age of breast cancer diagnosis in BRCA1 carriers was 42.0 (27-62) in BRCA2 carriers 43.7 (26-57), which was not significantly different. In $B R C A$ negative women it was 42.7 (26-64) years.

Of all the RRSO procedures $(\mathrm{N}=155)$, there were $141(91.0 \%)$ bilateral laparoscopic salpingooophorectomies, 5 (3.2\%) unilateral laparoscopic salpingo-oophorectomies and 7 (4.5\%) laparoscopic salpingectomies. One patient $(0.6 \%)$ had a bilateral and one had $(0.6 \%)$ a unilateral laparoscopic salpingo-oophorectomy.

\section{Pathological findings at RRSO}

Non-invasive or invasive serous high-grade cancer was diagnosed in 9 out of 155 (5.8\%) operated women (Table 1$)$. There were five $(3.2 \%)$ occult ovarian cancers and $4(2.6 \%)$ STICs. All cancers were detected among BRCA1 positive women as described in Table 1. Among STICs there were three BRCA1 carriers and one without a known mutation, but from the high risk group.

Among patients with occult invasive cancers, two were 39 years of age, all the other were older, mean age being 50.4 years. Mean age of patients with STICs was 57.8 years. The difference was not 
TABLE 1. Clinical characteristics of occult findings after RRSO

\begin{tabular}{|c|c|c|c|c|c|c|c|c|}
\hline $\begin{array}{l}\text { Patient } \\
\text { Number }\end{array}$ & $\begin{array}{c}\text { Age at RRSO } \\
\text { (years) }\end{array}$ & $\begin{array}{l}\text { Occult } \\
\text { finding }\end{array}$ & $\begin{array}{l}\text { BRCA gene } \\
\text { involved }\end{array}$ & $\begin{array}{c}\text { Type of pathogenic } \\
\text { variant }\end{array}$ & $\begin{array}{l}\text { FIGO } \\
\text { STAGE }\end{array}$ & Cytology & Treatment & $\begin{array}{c}\text { Vital } \\
\text { status }\end{array}$ \\
\hline 2 & 69 & STIC & BRCAl & c.3018_3021delTTCA & STIC & NEG & Surgery & NED \\
\hline 3 & 64 & STIC & negative/high risk & & STIC & NEG & Surgery & NED \\
\hline 4 & 45 & STIC & BRCAI & deletion exons 4-9 & $\begin{array}{c}\text { (Staging procedure } \\
\text { NEG) I C }\end{array}$ & PC & Surgery+ACT & NED \\
\hline 7 & 57 & HGSC & BRCAl & c.5266dup.C & IC & PC & Surgery+ACT & NED \\
\hline 8 & 39 & HGSC & BRCAl & C. $1687 C>T$ & III A & PC & Surgery+ACT & NED \\
\hline 9 & 61 & HGSC & BRCA 1 & deletion exons 4-9 & III A & PC & Surgery+ACT & OT \\
\hline
\end{tabular}

$\mathrm{ACT}=$ adjuvant chemotherapy; $\mathrm{DOOD}=$ died of other disease; FIGO = International Federation of Gynecology and Obstetrics; HGSC = high grade serous cancer; NED = no evidence of disease; NEG = negative; OT = on treatment; PC = peritoneal carcinomatosis; RRSO = risk reducing oophorectomy; STIC = serous intraepithelial tubal cancer

statistically significant. There were no occult cancers diagnosed among $B R C A 2$ carriers.

\section{Stages of cancer}

Three of four women with STIC underwent surgical staging procedure (Table 1). In three cases with STIC, staging procedure did not find any additional neoplastic cells and observation with no adjuvant treatment was recommended. In one case, the malignant cells were detected in the peritoneal cavity by cytological examination only. Stage was assessed to be I C. This patient received adjuvant chemotherapy with paclitaxel and carboplatin.

Among patients with occult cancers, one was assessed as stage I C, two as stage III A and two as stage III B. All received the adjuvant treatment with six courses of paclitaxel and carboplatin (Table 1).

\section{Localisation of findings}

Two STICs were found at the fimbrial part of fallopian tube and in the other two STICs, fallopian tube only was reported as a localisation. In invasive cancer patients, cancer cells were found in (i) two cases at the fimbrial part of the Fallopian tube and in one ovary, (ii) in one case the disease was present in both ovaries and in both Fallopian tubes, (iii) in one case cancer cells were present on the surface of both ovaries with normal Fallopian tubes and, (iv) in one case cancer cells were present on the surface of one ovary and in the Fallopian tube. Pathohistological findings included one patient with focally atypical epithelium of the fallopian tube with the addition of transitional cell metaplasia, one patient with adenomatoid hyperplasia with bilateral proliferation of Sertoli cells in both hiluses of ovaries that represented embryonal remnants and one patient with transitional cell metaplasia.

\section{CA-125 and cytology results}

All except one patient with STIC had a negative cytology result; on the other hand, all occult cancers had positive cytological findings.

Before the RRSO CA-125 measurement was performed in $83.9 \%$ of women. It was negative in all occult cancers and STICs except in one occult cancer, where it was slightly elevated, being $48 \mathrm{kU} / \mathrm{L}$ (normal value being $\leq 35 \mathrm{kU} / \mathrm{L}$ ). When considering CA-125 specificity in premenopausal years and normal vaginal ultrasound, patient was considered screen negative.

\section{Follow-up time of women with occult invasive and non-invasive cancer}

In our study the follow-up period was 23 to 73 months. Until December 2018 one woman with occult cancer died of gastric cancer which was diagnosed after adjuvant treatment for ovarian cancer. Two out of nine $(22.2 \%)$ are being treated for their third recurrence of disease, the rest $(6 / 9,66.7 \%)$ are alive with no signs of disease. Mean follow-up of patients with occult invasive and non-invasive ovarian cancer was 29 months (15-51). After RRSO, no woman developed peritoneal cancer. 


\section{Discussion}

We are presenting a population based study which aimed to address the prevalence, localization, type and outcome of occult cancer at RRSO in asymptomatic carriers of pathogenic or likely pathogenic BRCA1/2 variants and high-risk BRCA1/2 negative women.

Our main outcome was the detection of pathologic serous changes in tubes and ovaries in 5.8\% of all operated women.

At RRSO we found occult serous cancer in 5.5\% of $B R C A$ carriers, with $3.4 \%$ (5/145) having highgrade cancers and $2.1 \%$ (3/145) STICs; all were found in BRCA1 positive women. The prevalence of occult cancers in BRCA1 positive women in our study was $7.3 \%(8 / 110)$. According to the available literature, the prevalence of occult cancer found after RRSO varies from $2 \%$ to $17 \% .{ }^{13-22,28}$ In our study, the prevalence of occult cancer in BRCA1/2 positive women was $5.5 \%$, which is similar to what Conner et al. have found. ${ }^{29}$ We detected fewer occult cancers than Powell et al. who reported a rate of $7.9 \%$ and more than Reitsma et al. (2.2\%) and Finch et al. $(4.2 \%) .{ }^{14,16,24}$ It would be expected that studies that reported lower prevalence of occult cancers also had a lower median age at RRSO. Reitsma et al. reported the median age at RRSO to be 44 years of age, which is less than in our study where the median age at RRSO was $47 .{ }^{14}$ Since only asymptomatic women and screen negative women were included, the age at RRSO seems to be the most important factor which determines the higher prevalence of pathologic findings. When considering BRCA1/2 carriers, all pathological changes in our study were detected among the BRCA1 positive patients. In contrast, there were no pathological changes in 35 BRCA2 positive women. The speculative reason for this might be lower ovarian cancer penetrance and the later age of onset in BRCA2 carriers in comparison with $B R C A 1$ carriers and therefore critical number of cases for one case to be found was probably not achieved until the end of this evaluation.

Among high-risk BRCA negative women, the prevalence of occult serous disease was $10 \%(1 / 10)$. Limited data is available for the comparison of prevalence of occult cancers after RRSO in BRCA negative women. Only Reitsma's study from Netherlands found one (1/57) case of STIC and one $(1 / 57)$ case of atypical hyperplasia in RRSO specimens of BRCA-negative women with VUS. ${ }^{14}$ Our finding suggests the benefit and the importance of preventive surgeries in these women as well, though the number of our patients was very small.
An interesting and counterintuitive finding is the comparison of mean age among invasive and noninvasive cancers found at RRSO. Surprisingly, women diagnosed with STIC were older than women diagnosed with cancer. Mean age was 57.8 (45-69) and 50.4 (39-61) years, respectively. The difference was not statistically significant, most probably due to relatively small sample size. Furthermore, there was also insignificant trend of noninvasive cancer patients having breast cancer at an older age than those with invasive cancer. Similar findings were mentioned also in study from Powell et al. ${ }^{16}$

The localization of occult serous pelvic disease was coherent with the literature. Occult cancers were found in the tubal epithelium in $60 \%(3 / 5)$ and only in $40 \%(2 / 5)$ in the ovaries. In two cases the ovary was infiltrated only on the surface epithelium, in all other cases the cortex and stroma were also infiltrated. Intraepithelial serous lesions were found only in the tubal epithelium. In 50\% (2/4) of cases, STICs were found at the fimbrial part of tubes. In other two cases exact localization was not defined and is being revised. ${ }^{17}$

International Federation of Gynaecology and Obstetrics (FIGO) stage distribution among occult cancers diagnosed after RRSO was undoubtedly different and much more favourable than among population that presents with symptoms. There were $55.6 \%$ (4/9) of cancers staged I, II or in situ. There were no cancers staged higher than III B, two were III B and two were III A. The long term outcome is therefore expectedly better among patients diagnosed with ovarian/fallopian/peritoneal cancer after RRSO.

Cytological examination of peritoneal cavity washing was found to be very important. When all pathologic findings are negative, positive cytology finding is the only one that may determine further treatment. Women with positive cytology with all other specimens being negative are advised systemic chemotherapy based on cytology findings. ${ }^{26}$

Screening for ovarian cancer in the general population with CA-125 (and possibly transvaginal ultrasound) is generally not recommended due to its low sensitivity and specificity. It is, however, seen as a reasonable temporary alternative for women at high risk, who wish to delay RRSO. ${ }^{30}$ In our study, only one patient with an occult cancer had serum CA-125 levels above the cut-off value. CA-125 assay did not prove to be an effective screening tool for early cancer detection in our patients.

Genotype-phenotype correlations in BRCA carriers are not well defined and it is therefore difficult 
to estimate the exact risk of ovarian cancer associated with specific pathogenic variants. ${ }^{31}$ The mutational spectrum in patients with occult carcinoma in our study is in line with what is otherwise known about Slovenian BRCA carriers. Three of the detected variants are very common in Slovenian BRCA1 carriers, i.e. c.181T $>$ G p.(Cys61Ser), c.1687C $>\mathrm{T}$ p.(Gln563*) and c.5266dupC p.(Gln1756Profs $\left.{ }^{*} 74\right) .{ }^{32}$ In contrast, the deletion of exons 4-9 is only seen in $3.4 \%$ of our BRCA positive families but appears to be associated with a particularly high ovarian cancer risk. In this study, three out of eight (37.5\%) $B R C A$ positive patients with occult cancer carried this variant, which further supports the hypothesis that deletion 4-9 is highly penetrant with regards to ovarian cancer.

The main limitation of our study is already mentioned relatively small sample size that limits statistical evaluation. On the other hand, we were able to obtain an accurate clinical data for the sample studied. All studied women were tested and operated in our centre, where genetic testing and preventive follow up is performed on a national level.

\section{Conclusions}

In conclusion, a 5.8\% prevalence of occult invasive and noninvasive serous cancer after RRSO was found in high risk asymptomatic and screen negative $B R C A 1 / 2$ carriers. Most of occult invasive and noninvasive serous cancers were detected in $B R C A 1$ positive patients, yet the RRSO should also be considered as a preventive method for $B R C A$ negative high risk women. Age at preventive gynaecologic surgery should be carefully considered, taking into account the completion of childbearing age, and ideally, performed soon after 35, at least in BRCA1 patients. Cytology examination of peritoneal cavity washing should be performed in all cases of RRSO. And finally, our results favour the hypothesis of tubal origin of high-grade serous ovarian and peritoneal cancer.

\section{References}

1. Ferlay J, Colombet $M$, Soerjomataram I, Mathers C, Parkin DM, Piñeros $\mathrm{M}$, et al. Estimating the global cancer incidence and mortality in 2018: GLOBOCAN sources and methods. Int J Cancer 2019; 144:1941-53. doi: 10.1002/ijc.31937

2. Zadnik V, Primic Zakelj M, Lokar K, Jarm K, Ivanus U, Zagar T. Cancer burden in Slovenia with the time trends analysis. Radiol Oncol 2017; 51: 47-55. doi: 10.1515/raon-2017-0008
3. Kuchenbaecker KB, Hopper JL, Barnes DR, Phillips KA, Mooij TM, Roos-Blom $\mathrm{MJ}$, et al. Risks of breast, ovarian, and contralateral breast cancer for BRCA1 and BRCA2 mutation carriers. JAMA 2017; 317: 2402-16. doi: 10.1001/ jama.2017.7112

4. Mavaddat N, Peock S, Frost D, Ellis S, Platte R, Fineberg E, et al. Cancer risks for BRCA1 and BRCA2 mutation carriers: results from prospective analysis of EMBRACE. J Natl Cancer Inst 2013; 105: 812-22. doi: 10.1093/jnci/djt095

5. Antoniou A, Pharoah PDP, Narod S, Risch HA, Eyfjord JE, Hopper JL, et al. Average risks of breast and ovarian cancer associated with BRCA1 or BRCA2 mutations detected in case series unselected for family history: a combined analysis of 22 studies. Am J Hum Genet 2003; 72: 1117-30. doi: $10.1086 / 375033$

6. Kotsopoulos J, Gronwald J, Karlan B, Rosen B, Huzarski T, Moller P, at al. Agespecific ovarian cancer risks among women with a BRCA1 or BRCA2 mutation. Gynecol Oncol 2018; 150: 85-91. doi: 10.1016/j.ygyno.2018.05.011

7. Guidelines for assessment of ovarian cancer patients. [Slovenian]. Ljubljana: Institute of Oncology Ljubljana; 2016. [cites 2019 Nov 13]. Available from: https://www.onko-i.si/dejavnosti/zdravstvena-dejavnost/priporocila-inklinicne-poti/priporocila

8. Guidelines for diagnosis and treatment of breast cancer. [cites 2019 Nov 14]. Ljubljana: Institute of Oncology Ljubljana; 2018. Available from: https:// www.onko-i.si/fileadmin/onko/datoteke/Smernice/Smernice_diagnostike_ in_zdravljenja_raka_dojk_2018.pdf

9. Levanon K, Crum C, Drapkin R. New insights into the pathogenesis of serous ovarian cancer and its clinical impact. J Clin Oncol 2008; 26: 5284-93. doi: 10.1200/JCO.2008.18.1107

10. Nebgen DR, Hurteau J, Holman LL, Bradford A, Munsell MF, Soletsky BR, et al. Bilateral salpingectomy with delayed oophorectomy for ovarian cancer risk reduction: a pilot study in women with BRCA1/2 mutations. Gynecol Oncol 2018; 150: 79-84. doi: 10.1016/j.ygyno.2018.04.564

11. Leeper K, Garcia R, Swisher E, Goff B, Greer B, Paley P. Pathologic findings in prophylactic oophorectomy specimens in high-risk women. Gynecol Oncol 2002; 87: 52-6. doi: 10.1006/gyno.2002.6779

12. Callahan MJ, Crum CP, Medeiros F, Kindelberger DW, Elvin JA, Garber JE, et al. Primary fallopian tube malignancies in BRCA-positive women undergoing surgery for ovarian cancer risk reduction. J Clin Oncol 2007; 25: 3985-90. doi: $10.1200 / J C O .2007 .12 .2622$

13. Evans DG, Clayton R, Donnai P, Shenton A, Lalloo F. Risk-reducing surgery for ovarian cancer: outcomes and 300 surgeries suggest a low peritoneal primary risk. Eur J Hum Genet 2009; 17: 1381-5. doi: 10.1038/ejhg.2009.60

14. Reitsma W, De Bock GH, Oosterwijk JC, Bart J, Hollema H, Mourits MJE. Support of the "fallopian tube hypothesis" in a prospective series of riskreducing salpingo-oophorectomy specimens. Eur J Cancer 2013; 49: 132-41. doi: 10.1016/j.ejca.2012.07.021

15. Manchanda R, Abdelraheim A, Johnson M, Rosenthal AN, Benjamin E, Brunell $C$, et al. Outcome of risk-reducing salpingo-oophorectomy in BRCA carriers and women of unknown mutation status. BJOG An Int J Obstet Gynaecol 2011; 118: 814-24. doi: 10.1111/j.1471-0528.2011.02920

16. Powell CB, Swisher EM, Cass I, McLennan J, Norquist B, Garcia RL, et al. Long term follow up of BRCA1 and BRCA2 mutation carriers with unsuspected neoplasia identified at risk reducing salpingo-oophorectomy. Gynecol Oncol 2013; 129: 364-71. doi.: org/10.1016/j.ygyno.2013.01.029

17. Sherman ME, Piedmonte M, Mai PL, loffe OB, Ronnett BM, Van Le L, et al. Pathologic findings at risk-reducing salpingo-oophorectomy: Primary results from Gynecologic Oncology Group trial GOG-0199. J Clin Oncol 2014; 32: 3275-83. doi: 10.1200/JCO.2013.54.1987

18. Thompson C, McCormick C, Kamran W, O'Riain C, Norris L, Gallagher D, et al. Risk reduction surgery (RRS) for tubo-ovarian cancer in an Irish gynaecological practice: an analysis of indications and outcomes. Ir J Med Sci 2018; 187: 789-94. doi: 10.1007/s11845-017-1717-6

19. Ricciardi E, Tomao F, Aletti G, Bazzurini L, Bocciolone L, Boveri S, et al. Riskreducing salpingo-oophorectomy in women at higher risk of ovarian and breast cancer: a single institution prospective series. Anticancer Res 2017; 37: 5241-48. doi: 10.21873/anticanres.11948

20. Blok F, Dasgupta S, Dinjens WNM, Roes EM, van Beekhuizen HJ, EwingGraham PC. Retrospective study of a 16 year cohort of BRCA1 and BRCA2 carriers presenting for RRSO: Prevalence of invasive and in-situ carcinoma, with follow-up. Gynecol Oncol 2019; 153: 326-34. doi: 10.1016/j. ygyno.2019.03.003 
21. Zakhour M, Danovitch Y, Lester J, Rimel BJ, Walsh CS, Li AJ, Karlan BY, Cass I. Occult and subsequent cancer incidence following risk-reducing surgery in BRCA mutation carriers. Gynecol Oncol 2016; 143: 231-35. doi: 10.1016/j. ygyno.2016.08.336

22. Giannos A, Stavrou S, Douskos A, Drakakis P, Loutradis D. A salpingeal carcinoma revealed after prophylactic salpingoophorectomy in an asymptomatic BRCA1 carrier with breast malignancy. Int J Surg Case Rep 2018; 52: 107-10. doi: 10.1016/j.ijscr.2018.10.014

23. Clarke BA, Crum CP, Nucci, MR, Oliva MR. Protocol for the examination of specimens from patients with carcinoma of the fallopian tube. Based on AJCC/UICC TNM, 7th edition, and FIGO 2006 Annual Report. [cites 2019 Nov 15]. Available from: http://webapps.cap.org/apps/docs/committees/ cancer/cancer_protocols/2013/FallopianTube_13protocol_3101.pdf

24. Finch APM, Lubinski J, Møller P, Singer CF, Karlan B, Senter L, et al. Impact of oophorectomy on cancer incidence and mortality in women with a BRCA1 or BRCA2 mutation. J Clin Oncol 2014; 32: 1547-53. doi: 10.1200/ JCO.2013.53.2820

25. Domchek SM, Friebel TM, Singer CF, Evans DG, Henry T, Isaacs C, et al. Europe PMC funders group association of risk-reducing surgery in BRCA1 or BRCA2 mutation carriers with cancer risk and mortality. JAMA 2010; 304: 967-75. doi: 10.1001/jama.2010.1237

26. Patrono MG, Corzo C, Iniesta M, Ramirez PT. Management of preinvasive lesions. Clin Obstet Gynecol 2017; 60: 771-9. doi: 10.1097/ GRF.0000000000000316

27. Genetic/Familial High-Risk Assessment: Breast and Ovarian, version 3.2019 NCCN Guidelines, January 18, 2019. [cites 2019 Nov 15]. Available from https://www.nccn.org/professionals/physician_gls/default.aspx\#detection

28. Lavie O, Moskoviz MG, Auslender R, Gemer O, Bitterman A, Younes G, et al. Clinical and pathological characteristics of incidental diagnostic early occult malignancy after risk-reducing salpingo-oophorectomy in BRCA mutation carriers. Int J Gynecol Cancer 2016; 26: 233-9. doi: 10.1097/ IGC.0000000000000624

29. Conner JR, Meserve E, Pizer E, Garber J, Roh M, Urban N, et al. Outcome of unexpected adnexal neoplasia discovered during risk reduction salpingo-oophorectomy in women with germ-line BRCA1 or BRCA2 mutations. Gynecol Oncol 2014; 132: 280-6. doi: 10.1016/j.ygyno.2013.12.009

30. Drescher CW, Anderson GL. The yet unrealized promise of ovarian cancer screening. JAMA Oncol 2018; 4: 456-7. doi: 10.1001/jamaoncol.2018.0028

31. Rebbeck TR, Mitra N, Wan F, Sinilnikova OM, Healey S, McGuffog L, et al Association of type and location of BRCA1 and BRCA2 mutations with risk of breast and ovarian cancer. JAMA 2015; 313: 1347-61. doi: 10.1001/ jama.2014.5985

32. Stegel V, Krajc M, Zgajnar J, Teugels E, De Grève J, Hočevar $M$, et al. The occurrence of germline BRCA1 and BRCA2 sequence alterations in Slovenian population. BMC Med Genet 2011; 12: 9. doi: 10.1186/1471-2350-12-9 